Grzegorz Chromik*

Jagiellonen-Universität Krakau
UDK 811.112.2'28(438-13)

DOI: 10.4312/linguistica.60.2.45-63

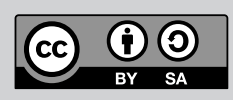

\title{
WILMESAU/WILAMOWICE - DIE NOCH EXISTENTE SPRACHINSEL IM SCHLESISCH-KLEINPOLNISCHEN GRENZRAUM UND DIE LEGENDE ÜBER IHREN NIEDERLÄNDISCHEN URSPRUNG
}

\section{EINLEITUNG}

Wilmesau, polnisch Wilamowice, ist eine Kleinstadt im Bezirk Bielsko-Biala in der Woiwodschaft Schlesien, Republik Polen. Sie war ab 1818 Marktgemeinde, zählt heute ca. 3000 Einwohner (Kacperczyk 2013: 131) und ist aktuell Hauptort einer Großgemeinde (Latosiński 1909: 118). Wilmesau hat eine eigene germanische Sprache und ist somit eine Sprachinsel innerhalb des polnischen Sprachraumes. Seit dem 18. Juli 2007 sieht die Internationale Organisation für Normung (ISO) innerhalb der Norm ISO 639-3, die Kennungen für Sprachen (Sprachkürzel, Sprachencodes, englisch language codes) definiert, einen Code für das dort gesprochene Wymysorys vor (,wym“) ${ }^{1}$. Diese Norm wird von der SIL International Organisation verwaltet.

\section{WAS IST WYMYSORYS?}

Man mag sich fragen, wie es möglich ist, dass eine polnische Kleinstadt ihre eigene Sprache hat. Wilmesau entstand im ausgehenden 13. Jahrhundert infolge der deutschrechtlichen Kolonisationswelle in diesem Gebiet. Als Ius Teutonicum wird die besondere Rechtsstellung der deutschen Siedler im Rahmen der mittelalterlichen Ostsiedlung in den vormals nichtdeutschen Gebieten Mitteleuropas bezeichnet. Dies beinhaltet eine weitgehende Ausnahme vom jeweiligen Landesrecht, insbesondere seinen Leistungspflichten und der Gerichtsbarkeit. Stattdessen wurde die Weiterführung der aus den ursprünglichen Siedlungsgebieten mitgebrachten Rechts- und Sozialordnung gewährt. Dieses Ius Teutonicum erscheint sowohl als Dorfrecht als auch als Stadtrecht (vgl. Menzel 1991). Charakteristisch dafür war die Gründung von großen Waldhufendörfern (Wilmesau ist räumlich gesehen auch eines) und deutschrechtlichen Städten im östlichen Mitteleuropa und die Ansiedlung von deutschen Bauern und Handwerkern.

Interessanterweise findet man in sehr vielen Werken die Information, dass die Wilmesauer nicht deutscher, sondern flämischer (auch: niederländischer, englischer) Abstammung seien. Davon ist auch die lokale Bevölkerung überzeugt und das „Wissen“ um diese „polnischen Flamen“ ist eigentlich in ganz Polen verbreitet. Der lokale Dialekt (oder, wie oben gesagt, die lokale Sprache) mag für einen Laien recht exotisch

gmchromik@yahoo.com

1 https://iso639-3.sil.org/code_tables/639/data/w?page=1 (15.09.2018). 
klingen, aber der Germanist wird beim ersten Hinhören stutzig. Dieser Beitrag verfolgt das Ziel, die Frage zu beantworten, wie viel Wahrheit im Mythos der niederländischen Abstammung der Wilmesauer steckt, bzw. dessen Genese zu analysieren. Wenn auch Wikipedia nicht als wissenschaftliche Quelle genutzt werden sollte, lässt sich die freie Enzyklopädie als Spiegel von Überlieferungen nutzen; sozusagen als Volksbuch des Internet-Zeitalters. Bis vor Kurzem wusste die deutschsprachige Wikipedia, dass der Ort ,im Zuge der Deutschen Ostsiedlung besiedelt [wurde]. Die Siedler sollen aus Flandern, Friesland, Holland und sogar Schottland gekommen sein" "2, obwohl die Sprache selbst ,vom Mitteldeutschen des 12. Jahrhunderts abgeleitet zu sein [scheint], allerdings mit einem starken niederdeutschen, niederländischen, polnischen und englischen Einfluss,“ denn ,[w]ahrscheinlich sind die Einwohner von Wilamowice Nachkommen von flämischen, deutschen und schottischen Siedlern, die Polen im 13. Jahrhundert besiedelten. ${ }^{\text {"3 }}$ Diese Beschreibung stellte das Wilmesaurische also als eine Mischung westgermanischer Sprachen dar. In der englischsprachigen Wikipedia heißt es immer noch: "The dialect is the result of the transformation of the Flemish language". Auf der Internetseite des Wilmesauer Magistrates steht:

\begin{abstract}
Das hiesige Gebiet gilt als Siedlungsgebiet der polnischen Flamen. Die Nachfahren der einstigen Siedler sind heute mit ihrer Sprache, ihrer schönen Tracht und dem Brauchtum eine einzigartige Kulturenklave. Die Geschichte von Wilmesau beginnt Mitte des 13. Jahrhunderts, als Herzog Wladislaus I. von Oppeln-Ratibor Siedler aus dem Westen in das von Tatareneinfallen verwüstete Land brachte. Die Siedler kamen wahrscheinlich aus Flandern und Friesland. ${ }^{5}$ [Übers. von G. Ch.].
\end{abstract}

Mittlerweile wurden die Angaben in der deutschsprachigen Wikipedia geändert. Die niederländisch-angelsächsisch-schottische Abstammung der Wilmesauer wird jetzt als Mythos bezeichnet. ${ }^{6}$ Man kann also hier die Kernfrage stellen: Ist der Wilmesauer Dialekt wirklich flämischer Herkunft oder doch nicht?

\title{
2 DER WILMESAUER DIALEKT
}

Dass die Einwohner des Ortes eine germanische Sprache sprachen - und manche noch sprechen - ist unumstritten. Die Sprache wird oft als Flämisch bezeichnet. Wilmesau liegt ca. $14 \mathrm{~km}$ vom Kern der ehemaligen deutschen Sprachinsel Bielitz entfernt. Eine flämische Sprachinsel in der Nähe einer deutschen wäre schon eine besondere Seltenheit. Glücklicherweise wird die im Aussterben begriffene Sprache immer noch verwendet, sodass neben der Sichtung alter dialektaler Texte auch die Befragung von Probanden möglich ist.

2 https://de.wikipedia.org/wiki/Wilamowice (15.09.2018).

3 https://de.wikipedia.org/wiki/Wilmesaurisch (15.09.2018).

4 https://en.wikipedia.org/wiki/Wilamowice (26.11.2018).

5 https://gmina.wilamowice.pl/strona/o-gminie (26.11.2018).

6 https://de.wikipedia.org/wiki/Wilamowice\#cite_note-w380-10 (08.02.2020). 


\subsection{Die Verschriftlichung des Dialektes}

Seit dem 19. Jahrhundert ist es in Wilmesau üblich, den Dialekt zu verschriftlichen. Es gibt mehrere Rechtschreibungen und eigentlich schrieb jeder Autor anders. Eine in Wilmesau allgemein anerkannte Norm gibt es erst seit 2009. Alle Wilmesauer Schreibungen zeichnen sich dadurch aus, dass für die dem Deutschen unbekannten Laute polnische Schriftzeichen $s$ und $t$ verwenden. Diese Laute unterscheiden das Wilmesaurische (neben zahlreicheren Polonismen im Wortschatz) von anderen Orten der deutschen Sprachinsel Bielitz. Darüber hinaus werden polnische Schreibsitten den deutschen vorgezogen (etwa $c$ und nicht $z$ für [ts], $h$ für [x], $y$ für [I]).

\subsection{Die Wenkersätze und andere Beispiele des Wilmesauer Dialekts}

Zur Darstellung eines deutschen Dialekts eignen sich die Wenkersätze. ${ }^{7}$ Bei jedem Satz findet man die Wilmesauer Version und zum Vergleich die Übersetzung in die niederländische Standardsprache (Vragenlijsten met register (1931-1958) 1960: 3-5). Im Folgenden werden die ersten fünf übersetzten Wenkersätze zitiert:

1. Im Winter fliegen die trocknen Blätter durch die Luft herum.

Ym wynter fligja dy dyra błytyn yr töft rym.

In den winter vliegen de droge bladeren door de lucht.

2. Es hört gleich auf zu schneien, dann wird das Wetter wieder besser. S'hjyt bałd uf cy śnäjn, do wjyd dy cäjt wejder beser.

Het houdt dadelijk op met sneeuwen, dan wordt het weer wel weer beter.

3. Tu Kohlen in den Ofen, dass die Milch bald an zu kochen fängt.

Łe köła yn uwa, do dy mytih gryht oziöet.

Doe (wat) kolen op de kachel, dat de melk gauw gaat koken.

4. Der gute alte Mann ist mit dem Pferde durchs Eis gebrochen und in das kalte Wasser gefallen.

Der güty, ołdy kłop ej mytum fad ym äjs äjgybroha àn a ej ys kotdy woser nájgyfoła.

De goede oude man is met zijn paard door het ijs gezakt en in het koude water gevallen.

5. Er ist vor vier oder sechs Wochen gestorben.

A ej śun fjyr oba záhs woha y dam gyśtiörwa.

Hij is voor vier of zes weken gestorven.

7 Wenkersätze nach regionalsprache.de, Datei 17738a, in modernisierter Schreibung (Änderungen des Muttersprachlers Tymoteusz Król aus Wilmesau). 
Ein besonderes Beispiel des Wilmesaurischen ist auch in einem Abschnitt aus einem literarischen Text in dieser Sprache zu lesen: Florian Biesik, ein aus Wilmesau stammender und Jahrzehnte lang in Triest wohnhafter k. u. k. Postbeamter, verfasste 1921 eine Überarbeitung der „Göttlichen Komödie“ von Dante im Dialekt seiner Heimat. ${ }^{8}$

\begin{tabular}{|l|l|l|l|}
\hline & Wilmesaurisch & Deutsch & Niederländisch $^{9}$ \\
\hline 1 & $\begin{array}{l}\text { Fylón yr wett an ganc } \\
\text { attán }\end{array}$ & $\begin{array}{l}\text { Verloren in der Welt und } \\
\text { ganz allein }\end{array}$ & $\begin{array}{l}\text { Verloren in de wereld en } \\
\text { gans alleen }\end{array}$ \\
\hline 2 & $\begin{array}{l}\text { zas ych óm mjer óf hóhym } \\
\text { śtán; }\end{array}$ & $\begin{array}{l}\text { Saß ich auf dem Meer auf } \\
\text { hohem Stein }\end{array}$ & $\begin{array}{l}\text { zat ik aan zee op een } \\
\text { hoge steen }\end{array}$ \\
\hline 3 & $\begin{array}{l}\text { der mond kąm raus } \\
\text { hyndróm gybjég, }\end{array}$ & $\begin{array}{l}\text { Der Mond kam raus hinter } \\
\text { dem Gebirge }\end{array}$ & $\begin{array}{l}\text { de maan kwam van ginder } \\
\text { om de berg }\end{array}$ \\
\hline 4 & $\begin{array}{l}\text { szłyfyt myjch áj, wi s kynt } \\
\text { yr wig. }\end{array}$ & $\begin{array}{l}\text { Schläfert mich ein, wie } \\
\text { ein Kind in der Wiege }\end{array}$ & $\begin{array}{l}\text { slaap-voerde mij af, als } \\
\text { een kind in de wieg }\end{array}$ \\
\hline
\end{tabular}

Das grammatischen Paradigmen nach Latosiński (1909: 273f.) sehen folgendermaBen aus:

Deklinationsparadigma:

\begin{tabular}{|l|l|l|}
\hline & Singular & Plural \\
\hline Maskulinum (Schüler & dyr siter & dy sityn \\
,Lehrer') & s sityś & dyr sityn \\
& ym siter & yn sityn \\
& a siter & dy sityn \\
\hline Femininum (Sister & $d y$ zyster & dy zystyn \\
,Schwägerin') & s zystyś & dyr/dar zystyn \\
& yr zyster & yn zystyn \\
& dy zyster & dy zystyn \\
\hline Neutrum (Schwalbe) & s śwaumta & dy śwaumta \\
& s śwaumta & dyr śwaumta \\
& ym śwaumta & yn śwaumta \\
& s śwaumta & dy śwaumta \\
\hline
\end{tabular}

8 http://bielskobiala.wyborcza.pl/bielskobiala/7,88025,21496653,of-jer-welt-przedstawienie-powilamowsku-na-deskach-teatru.html (26.11.2018).

9 https://news.ycombinator.com/item?id=17277691 (26.11.2018). 
Das Verb:

Konjugationsparadigmen (Präsens):

\begin{tabular}{|c|c|c|c|c|c|}
\hline & & sein & haben & $\begin{array}{l}\text { schwaches } \\
\text { Verb (legen) }\end{array}$ & $\begin{array}{l}\text { starkes Verb } \\
\text { (backen) }\end{array}$ \\
\hline Singular & $\begin{array}{l}Y h \\
d u / d y \\
\operatorname{har}(a ́) / z y j / s\end{array}$ & $\begin{array}{l}\text { byj } \\
\text { byst } \\
y j\end{array}$ & $\begin{array}{l}\text { ho } \\
\text { höst } \\
\text { höt }\end{array}$ & $\begin{array}{l}\text { tee } \\
\text { teest } \\
\text { teet }\end{array}$ & $\begin{array}{l}\text { Bak } \\
\text { bekst } \\
\text { bekt }\end{array}$ \\
\hline Plural & $\begin{array}{l}W y r \\
j y r \\
z y / z y j\end{array}$ & $\begin{array}{l}\text { zaein } \\
\text { zaeit } \\
\text { zaein }\end{array}$ & $\begin{array}{l}\text { honn } \\
\text { hott } \\
\text { honn }\end{array}$ & $\begin{array}{l}\text { teen } \\
\text { teet } \\
\text { teen }\end{array}$ & $\begin{array}{l}\text { Baka } \\
\text { bakt } \\
\text { baka }\end{array}$ \\
\hline
\end{tabular}

Konjugationsparadigmen (Perfekt):

\begin{tabular}{|l|l|l|l|l|l|}
\hline & sein & haben & $\begin{array}{l}\text { schwaches Verb } \\
\text { (legen })\end{array}$ & $\begin{array}{l}\text { starkes Verb } \\
\text { (backen })\end{array}$ \\
\hline Singular & Yh & byj gywast & ho gyhot & ho gyteet & ho gybaka \\
\hline
\end{tabular}

Es ist natürlich unmöglich, eine Sprache in einem kurzen Beitrag eingehend darzustellen.

Deswegen wird sie hier nur ganz kurz besprochen, indem als Grundlage die deutsche Standardsprache angenommen und auf die wichtigsten Unterschiede hingewiesen wird.

\subsection{Analyse der Dialektproben}

Der Dialekt weist konsequent die hochdeutsche zweite Lautverschiebung auf: zas ych vs. niederländisch zat ik, of vs. op; szłyfyt-slaap, Kap. 2.2 - Wenkersätze). Nimmt man die in der Germanistik als Grundlage angenommene Klassifizierung der germanischen Sprachen, nach welcher die zweite Lautverschiebung das Kriterium für die Zuordnung eines Dialekts zum Hochdeutschen ist, so muss das Wilmesaurische als Hochdeutsch bezeichnet werden, was Zweifel an der volkstümlichen Theorie der niederländischen (flämischen) oder englischen Herkunft der Wilmesauer aufkommen lässt. Die sonstigen westgermanischen Sprachen entwickelten die zweite Lautverschiebung nicht, was auch für die niederdeutschen Dialekte gilt (Stedje 1989: 59).

Auch die restliche Analyse des sprachlichen Materials lässt keinen Zweifel offen das sog. Wilmesaurisch ist ein hochdeutscher Dialekt. Vom Niederdeutschen oder Niederländischen, geschweige denn Englischen oder „Schottischen“, fehlt jede Spur.

\subsubsection{Lautlehre}

Nach phonologischen Kriterien ist Wilmesaurisch eine deutsche Mundart. Der Vokalismus entspricht dem Ostmitteldeutschen: die Diphthongierung und Monophthongierung treten auf (vgl. die Wenkersätze - etwa ftigja, güty, bridyn [,Brüder'], ajs, broüny (39), łoüt). Eine Ausnahme ist die Präposition uf: Die zweite Lautverschiebung entspricht ebenfalls den ostmitteldeutschen Gesetzmäßigkeiten (sie fehlt im Auslaut 
und Inlaut intervokalisch bei $p$ - also töp [,Topf'], hop [,Hopfen'], tepper [,Töpfer'], troppa [,Tropfen'], im Anlauf erscheint $f$ statt $p f$ : fund [,Pfund'], Fyngsta [,Pfingsten'], fad [,Pferd'], im Auslaut nachkonsonantisch $f$ wie in der Schriftsprache: duyf [,Dorf'], siuöf [,scharf‘] (Latosiński 1909: 284, 287, 291, 292, 299, 314, 318). Die Lautverschiebung entspricht bei $t$ und $k$ in der Aussprache der deutschen Standardsprache, in der Schrift verwendet man die Zeichen $c$ statt $(t) z$ und $h$ statt $c h$.

Schon das oben Gesagte genügt für die Widerlegung des Flamen-Mythos, denn die niederländischen Dialekte haben nicht an der zweiten Lautverschiebung teilgenommen. Das obige sprachliche Material liefert aber mehr. Auch andere, für das Niederländische typische Laute sind nicht vorhanden und entsprechen den deutschen Lauten. Genauso entsprechen die Personalpronomina den (mittel)deutschen Formen und nicht den niederländischen, wie etwa

$f$ in töft ,Luft' wie im Dt. (nicht $f$ wie im Nl. lucht); har (heute eher $a$ ), nicht hij; $t$ im Anlaut wie im Dt. (statt des nl. d): tün vs. nl. doen; Personalpronomen ihr (3. P. Sg., Dat. Fem.): jyr, ohne $h$ (wie im nl. haar, englisch her); es: $s$ (ohne $h$ wie im nl. het); $d u$ wie im Dt. (nicht jou); wyr mit $r$ wie im Dt. (nicht we). ${ }^{10}$

Das Wilmesaurische weist allerdings einige phonetische Eigenschaften auf, die der niederländischen Standardsprache und den mitteldeutschen Dialekten, jedoch nicht der deutschen Standardsprache entsprechen: stimmhaftes $d$ statt $t$ nach Liquida: ołdy, kołdy, wutda (dt. alte, kalte, wollten, nl. oude, koude, wilden) u. a. ${ }^{11}$

Alle oben dargestellten Erscheinungen sind oder waren früher in den mitteldeutschen Dialekten bekannt (dazu vgl. Chromik 2010: 70, 73, 112, Chromik 2017: 177, Reichmann/Wegera 1993: 45, 49, 106, 310, Rückert 1878: 193).

10 Dies gilt auch für weitere Lautverbindungen wie etwa: schl/schn in gyśtyfa, śnej ,Schnee“ (wie im Dt., nicht sl/sn wie nl. slecht, geslaap, sneeuw); re in drescha (wie im Dt., nicht or wie nl. dorschen); $k s$ in öksa 'Ochsen' (nicht [s] wie im Nl.) vs. nl. ossen.

11 Z. B. Senkung von e zu a: fad (vs. dt. Pferd, nl. pard); Konjunktion en 'und' ohne [t] im Auslaut: an (vs. dt. und, nl. en); [v] statt [b] im Inlaut: gyśtiörwa, liw, öwyts (vs. dt. gestorben, lieb, abends, nl. gestorven, lieve, avond); Part. Pf. von sein: gewast (vs. dt. gewesen, nl. geweest); fehlende zweite Lautverschiebung von $p$ zu $p f$ im Inlaut, z. B. apeła (vs. dt. Äpfelchen, nl. appeltjes). 


\subsubsection{Wortschatz}

Auch der Wortschatz weist viele typische schlesische Wörter auf. Im Folgenden wird nur eine Auswahl angegeben. Die schlesischen Vergleichsbelege reichen bis ins Spätmittelalter:

\begin{tabular}{|l|l|l|}
\hline Wilmesaurisch & $\begin{array}{l}\text { deutsche } \\
\text { Standardsprache }\end{array}$ & $\begin{array}{l}\text { schlesisches } \\
\text { Frühneuhochdeutsch }\end{array}$ \\
\hline kuza & Sprechen & kusa $^{12}$ \\
\hline Nökwer & Nachbar & Nokwer $^{13}$ \\
\hline of, uf & auf & of, uf ${ }^{14}$ \\
\hline Puś & Wald & Posch \\
\hline Rynk & Marktplatz & Ring \\
\hline Zaegier & Uhr (Zeiger) & Zeiger \\
\hline Fuyt & Dorfschulze (Vogt) & Voyt \\
\hline
\end{tabular}

Das belegt eindeutig, dass die Wilmesauer Sprache (oder Mundart) keine anderen Sprachspuren aufweist als schlesisches Deutsch. Die einzigen nichtdeutschen Merkmale sind Polonismen, was aber bei einer Sprachinsel inmitten des polnischen Sprachraumes nicht wundert und nicht Thema dieses Beitrags ist. Ursächlich für die Konservierung des Dialekts und den im Folgenden dargestellten Schwund des ,deutschen Bewusstseins" waren die verlorengegangene Anbindung an andere Dörfer des östlichen Teils der Sprachinsel und die Abschaffung des deutschen Schulwesens im 19. Jahrhundert.

Die Abb. 1 zeigt, dass die Polonisierung der Dörfer Wilmesdorf/Stara Wieś und Pisarzowice/Schreibersdorf sowie die Auswanderung der deutschen Bevölkerung aus Seibersdorf/Kozy nach Preußen 1770 das Dorf Wilmesau zu einer Exklave der großen deutschen Kernsprachinsel machte. All dies war ein guter Boden für das Aufkommen des Mythos über eine exotische Herkunft der Einwohner.

12 Vgl. „Sprechen Sie Karpatendeutsch“ Dokumentarfilm über die karpatendeutsche Minderheit in der Slowakei. Regie: Jozef Tancer und Anna Grusková (2018). Ursprünglich kôsen: ,gespräch führen, sprechen, plaudern' http://woerterbuchnetz.de/cgi-bin/WBNetz/wbgui_ py?sigle $=$ Lexer\&mode=Vernetzung\&lemid $=$ LK02768\#XLK02768 (26.11.2018).

13 Belegt in Krossen/Krosno (Kleinpolen) 1462. Vgl. Chromik (2016: 113).

14 Rückert (1878: 109f.). 
Abb. 1: Deutsche Sprachinsel Bielitz vor der Vertreibung 1945. Dunklere Farbe zeigt deutsche Dörfer, hellere polnische ${ }^{15}$ :

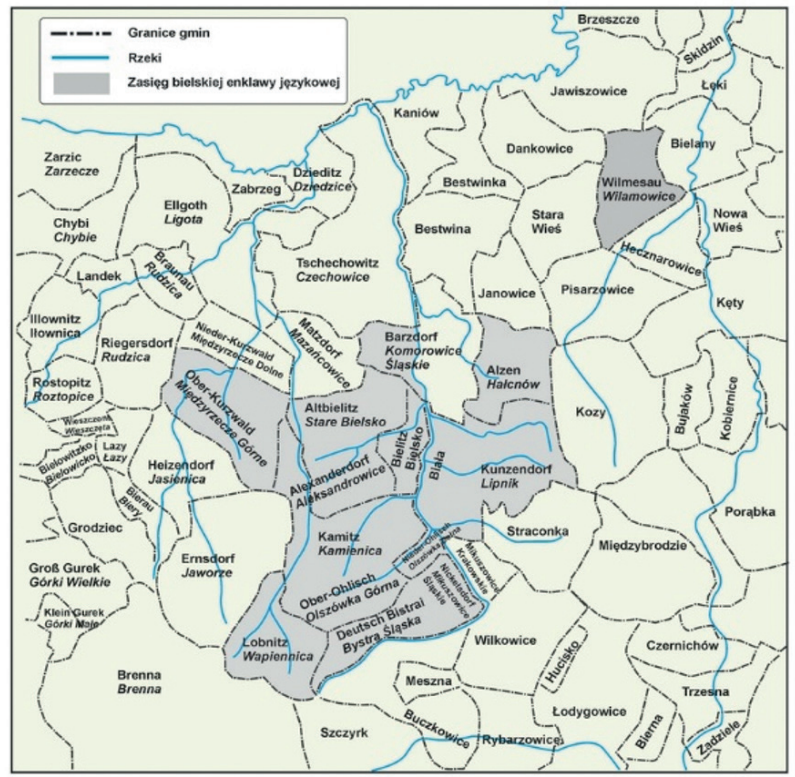

\subsection{Trennung vom Rest der deutschen Sprachinsel Bielitz}

Geographisch betrachtet ist Wilmesau im Grunde eine kleine, separierte Sprachinsel unweit der großen Bielitzer Sprachinsel. Der Grund dafür ist die Polonisierung der Wilmesau mit der Kernsprachinsel verbindenden Dörfer Stara Wieś/Wilmesdorf und Pisarzowice/Schreibersdorf. Die Polonisierung dieser Dörfer ist ein Thema für weitere Untersuchungen. Auf diese Weise wurde Wilmesau zu einer winzig kleinen Sprachinsel, was besondere sprachliche, vor allem phonetische Entwicklungen mit sich brachte (siehe Abschnitt 2.3).

\section{DAS BILD DER WILMESAUER UND IHRER SPRACHE IN DER GESCHICHTE}

\subsection{Das 17. und 18. Jahrhundert}

Die älteste Erwähnung der ethnischen Verhältnisse in Wilmesau wurde 1640 vom lokalen (polnischen) Pfarrer in der ältesten erhaltenen Kirchenmatrikel verfasst:

Im Jahre 1640, am neunten Tage des Monats August, als der Hochwürdige Herr Grzegorz Jan Zdziewojski, Prediger von Auschwitz, Doktor der Sieben Freien Künste und der Philosophie, fünfzehn Jahre nach der Häresie die Pfarrei Wilmesau übernahm, die hundert Jahre unter der Sekte Calvins herrschte, hatte diese

15 Bild von: Dziedzictwo Kulturowe Rzeczypopolitej [Kulturerbe Polens] http://www.inne-jezyki. amu.edu.pl/Frontend/Language/Details/10 (04.12.2018). 
Kirche keinen eigenen Pfarrer und wurde nur von Verwaltern geleitet, sowohl von Weltpriestern als auch von Ordensbrüdern, weil es keine der deutschen Sprache kundigen Priester gab und hier in diesem Dorfe Wilmesau das deutsche Volk wohnte. Deswegen blieben die Priester nur einige Sonntage da, die Kirche war arm und die Leute kümmerten sich nicht um die Ausstattung der Kirche. ${ }^{16}$ [Übers. aus dem Poln. von G. Ch.]

In dem bekannten Buch von Latosiński (1909: 173) wird ein Gedicht in polnischer Sprache angeführt, das aus Anlass der Fertigstellung des Wilmesauer Kirchturmes im Jahr 1729 verfasst wurde und das als Beweis für das fehlende Zugehörigkeitsgefühl der Wilmesauer zum Deutschtum gelten soll. Es handelt sich um den Ausdruck [...] majstra Niemca my mieli [,wir hatten einen deutschen Meister"]. Dieser Beweis ist aber sehr fraglich, da das Gedicht zum einem wahrscheinlich vom Wilmesauer Pfarrer Stanisław Sienieński, einem Polen, verfasst wurde, und andererseits ist es auch möglich, dass es sich beim obigen Ausdruck nicht um die Nationalität, sondern um einen Familiennamen handelt. In den bischöflichen Visitationsberichten findet man keine Angaben zur Predigtsprache.

\subsection{Das 19. Jahrhundert}

Die Urkundensprache der Gemeinde war bis zum Beginn des 19. Jahrhunderts Polnisch. In den Jahren 1817-1820 erschienen neben polnischen Urkunden auch deutsche, und ab 1826 nur noch deutsche. Aber bereits 1885 wurde die Verhandlungs- und Protokollsprache der Gemeindeverwaltung wieder Polnisch ${ }^{17}$. Diese Wechsel waren wahrscheinlich durch die Präferenzen der feudalen Obrigkeit bedingt (Latosiński 1909: 122f.), die durchgehend polnisch war. Nach den napoleonischen Kriegen wurden die Germanisierungstendenzen stärker, wodurch der Gebrauch der deutschen Amtssprache in Galizien gestärkt wurde. Der letzte Wechsel zum Polnischen war eine Entscheidung einer neu gewählten Gemeindevertretung, die zwar aus einheimischen Mundartsprechern bestand (Bürgermeister Franz Schneider), aber wahrscheinlich dem Sprachprestige des Polnischen in Galizien folgte. Nicht auszuschließen ist auch der Einfluss des dortigen Schullehrers und überzeugten polnischen Patrioten Józef Latosiński (Latosiński 1909: 137f.).

Im frühen 19. Jahrhundert hielt man den Wilmesauer Dialekt für Deutsch: „Die Gemeinde Wilamowice hat das sonderbare, daß sich Deutsch in der Sprache erhalten hat seit der Urzeit, obwohl ringsum polnisch gesprochen wird“"(Augustin 1842) ${ }^{18}$.

16 Metrika Baptisatorum ab ao 1641, f. 1.

17 Morciniec, Norbert: Vom Sterben einer Mundart. http://www.morciniec.eu/79,vom_sterben einer_mundart (03.12.2018).

18 Augustin, Franz: Jahrbuch oder Zusammenstellung geschichtlicher Thatsachen, welche die Gegend von Oswieczyn und Saybusch angehen. Bearbeitet durch Franz Augustin Pfarrer der Stadt Saybusch 1842 ad A R D Andrea de Pleszowski porocho Bielanensi propria namu descriptum. Archchiwum Państwowe w Katowicach, Oddział Żywiec, S. 166. Zugänglich auf: http://innejezyki.amu.edu.pl/Editor/files/Wilamowice\%20u\%20Augustyna\%20166-167.jpg (03.12.2018). 
Wann die Ansicht über die abweichende Herkunft der Wilmesauer entstand, wissen wir heute nicht. Etwas Licht ins Dunkel wirft die 1860 herausgegebene Sammlung der Volksdichtung aus der deutschen Sprachinsel Bielitz von Jacob Bukowski „Gedichte in der Mundart der deutschen schlesisch-galizischen Gränzbewohner, resp. von BielitzBiala“. Das Gedicht $A$ Welmeßajer ai Berlin erzählt von den Verständigungsproblemen eines Wilmesauer Kaufmanns in Berlin:

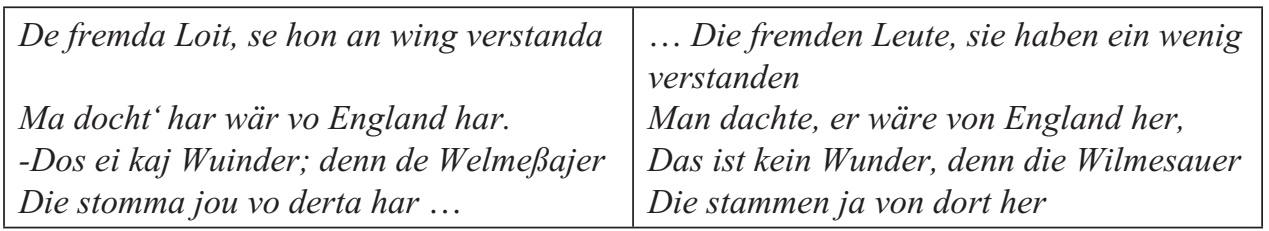

Mitte des 19. Jahrhunderts dürfte der Wilmesauer Dialekt bereits in der Stadt Bielitz befremdlich gewirkt haben. Man orientierte sich an der Schriftsprache und entlehnte sogar „wienerische“ Dialektalismen (Bock 1916: 7). Englisch war kaum bekannt und auch andere deutsche Dialekte kannte man wenig. Ein dem Wilmesaurischen ähnlicher Dialekt (des knapp zehn Kilometer entfernten Alzen) wurde in Bielitz als „Schwäbisch“ bezeichnet (Huppert 1976: 52, 76-77). Da ist der Weg zu flämischen Legende nicht mehr weit: Jeder realistische Ahnenforscher weiß, dass eine exotische Abstammung (unterstützt durch Volksetymologie von Familiennamen) sich sehr hartnackig halten kann. Und ebenso groß die Enttäuschung, wenn sich das als bloße Legende entpuppt.

Bis zum Ausgang des 18. Jahrhunderts gebrauchten die Einwohner von Wilmesau in der Kirche ihre eigene Mundart. Als sich die Gemeinde im Jahre 1808 vom Frondienst freikaufte und der Zustrom polnischer Bevölkerung begann, wurden auch polnische Gottesdienste eingeführt. Seit 1829 gab es in der Kirche keinen deutschen Pfarrer mehr. ${ }^{19}$ Es ist anzunehmen, dass bis 1875 in der Wilmesauer Schule, ungeachtet der Tatsache, dass sie offiziell polnisch war, in lokaler Mundart unterrichtet wurde, zumal alle Lehrer Einheimische waren. Dies war allerdings eine Ausnahmeerscheinung im offiziell polnischsprachigen Kronland Galizien. Diese Information stammt von einem Lehrer selbst, ist also ziemlich glaubwürdig. Auf die einheimischen Lehrer folgten dann ethnische Polen aus anderen Gegenden Galiziens, die natürlich die Mundart nicht beherrschten und die Kinder auf Polnisch unterrichten mussten (Latosiński 1909: 264f.). Eine zweite deutsche Schule wurde in Wilmesau erst 1912 eingerichtet, doch besuchten sie in diesem Jahr nur 80 von 300 Schülern. Offenbar sahen die meisten Eltern ein besseres Fortkommen ihrer Kinder in einem Besuch der polnischen Schule. Im häuslichen Verkehr jedoch wurde weiterhin die Wilmesauer Mundart gesprochen. Die Popularität des Polnischen soll nicht verwundern: In den letzten Jahren der k. u. k. Monarchie erlebte das Polnische in Galizien, dessen Teil Wilmesau ja war, eine Blütezeit.

19 Morciniec, Norbert: Vom Sterben einer Mundart http://www.morciniec.eu/79,vom_sterben_einer_ mundart (03.12.2018). 


\title{
3.3 Das 20. Jahrhundert - die Blütezeit der Legenden
}

Zur Verbreitung der allgemeinen Überzeugung, die Wilmesauer seien flämischer Abstammung, trugen mit Sicherheit zwei Publikationen bei, die 1907 und 1909 erschienen. Diese Ausführungen zu sprachlichen Merkmalen des lokalen Dialekts sind wissenschaftlich sehr umstritten.

Im Jahr 1907 veröffentlichte der lokale Schullehrer Ludwik Młynek, der selbst aus Tarnów in Galizien stammte, ein Büchlein mit dem Titel „Narzecze wilamowickie (Dy wymmysuarischy Gmoansproch)“. Zur Herkunft der Wilmesauer gab er die vor Ort kursierenden Legenden wieder. Die einen sahen ihre Vorfahren als Alemannen, die anderen als Sachsen oder gar Angelsachsen. Dass sich diese Hypothesen gegenseitig völlig ausschließen, interessierte ihn wenig. Als einzigen Beweis der englischen Abstammung wurden die häufigsten Familiennamen angegeben - Foks, Figwer, Danek (Młynek 1907: 8-10). Die Ähnlichkeit des Familiennamens Fox/Foks mit dem englischen Substantiv fox ist aber auch auf der Grundlage des Schlesischen zu erklären - die Senkung des $u \mathrm{z} \mathrm{u} o$ war im älteren Schlesisch typisch. Figwer ist ein deutscher Name (Viehweger), trat früher nicht nur in Wilmesau auf (Kuhn 1981: 94f.), und Danek wohl polnisch.

Konsequenter war der Autor der in Wilmesau seit über hundert Jahren beliebten Monographie Józef Latosiński (1909). Hier heißt es auf den Seiten 13 und 14 (übers. aus dem Poln. von. G. Ch.):

\begin{abstract}
Will man nachweisen, woher die ersten Wilmesauer kamen, so muss man an den örtlichen Dialekt denken, der dabei der sicherste Hinweis ist. Der Kern des Wilemsauer Dialekts [...] ist die althochdeutsche und mittelhochdeutsche Mundart, darüber hinaus sieht man die Spuren des Gotischen, der niederdeutschen Mundarten (Angelsächsisch, Altnordisch usw.) sowie der holländischen und englischen Sprache. [...] Die historischen Quellen weisen darauf hin, dass Mieczyslaus, der Herzog von Oppeln und Ratibor, Siedler überwiegend aus dem Herzogtum Schaumburg-Lippe nach Bielitz und in die Umgebung brachte [...]. Die Lage des Herzogtums Schaumburg-Lippe, das unweit der holländischen Grenze und Bremens liegt, weist darauf hin, dass die ersten Wilemsauer von dort kamen. Das erstere wegen des lokalen Dialekts, der neben dem angestammten niederdeutschen Kern auch holländische und angelsächsische Merkmale aufweist, das andere deswegen, weil im Herzogtum Schaumburg-Lippe bis heute die Weberei floriert und eben die Weberei zur Grundlage des Wohlstandes der Wilmesauer wurde und bis heute eine große Rolle spielt.
\end{abstract}

Dies strotzt vor Widersprüchen: einerseits sei der Kern der Mundart althoch-, andererseits gleichzeitig niederdeutsch.

Nach Wicherkiewicz lieferte der bereits erwähnte Wilmesaurische Autor Florian Biesik (1850-1926) die meisten Argumente für eine nichtdeutsche Herkunft der Wilmesauer. In seiner Dichtung Wymmysau an wyjmysojer schrieb er: 
Vertryjn wegia religion

polityk an rebellion

wandytas fy England, Holland

an kooma bocy óf Póttand
Vertrieben wegen Religion

Politik und Rebellion

wanderten aus England, Holland aus

und kamen sogar nach Polen

Selbst sein Bruder, Hermann Mojmir, glaubte nicht an diese Mythen. Er hielt den Wilmesauer Dialekt für typisch schlesisch, was wahrscheinlich auf seine persönlichen Kontakte zu Krakauer Germanisten zurückzuführen ist. 1920/1921 erschien sein zweibändiges Werk Dialekt Wilamowic w zachodniej Galicji [,Der Dialekt von Wilmesau in Westgalizien']. Unter der Redaktion von Kleczkowski erschien in Jahren 1930-1936 sein Wörterbuch der deutschen Mundart von Wilamowice. Nach dem Erscheinen dieser Werke ruhten die Untersuchungen des Wilmesauer Dialekts einige Jahrzehnte lang, bis in die 1980er Jahre. Dazwischen liegt die Katastrophe des Zweiten Weltkrieges, die auch der kleinen Wilmesauer Kultur ihr Ende brachte, wenn auch auf eine ganz andere Weise als im Fall der Kernsprachinsel, deren deutsche Einwohner einfach vertrieben wurden. Die Wilmesauer beriefen sich bei der sowjetischen Militärverwaltung darauf, dass die Bestimmungen der Potsdamer Konferenz Deutsche betrafen, nicht aber andere nichtpolnische Volksgruppen, etwa Nachfahren der Flamen und Niederländer, die sie ja seien. Tatsächlich wurde ihnen der Verbleib erlaubt, wobei die polnischen Behörden ,,vorsichtshalber" den Gebrauch des Dialekts untersagten. Der bekannte Forscher zur Geschichte der deutschen Bevölkerung in den Beskiden, Walter Kuhn, kommentierte dies wie folgt:

\begin{abstract}
Sogar eine schottische Beimischung wird ihnen nachgesagt, die Gründe dafür sind das Vorkommen des Namens Fox in Wilmesau und die Deutung des Lokatorennamens Wilhelm als William, im Anschluß an die polnische Form des Dorfnamens Wilamowice. So kurios diese Theorien auch sind und so sehr sie mit den Tatsachen, vor allem der rein schlesischen Dorfmundart, im Widerspruch stehen, so bilden sie doch einen gewissen Schutz für dieses heute einzige deutschsprachige Dorf in Polen [Kuhn 1981: 53].
\end{abstract}

In den 1980er Jahren beschäftigte man sich wieder mit dem Wilmesauer Dialekt. Norbert Morciniec verfasste 1984 den Beitrag „Die flämische Ostkolonisation und der Dialekt von Wilamowice in Südpolen”. Im selben Jahr schrieb der flämische Dialektologe Hugo Ryckeboer einen Beitrag, im dem das Wort „Flamen“ in Anführungsstrichen stand (Wicherkiewicz 2013: 5).

Deutsche Dialektologen betrachten Wilmesaurisch, zusammen mit der gesamten deutschen Sprachinsel Bielitz und den isolierten Dörfern Kosthenthal/Gościęcin und Schönwald/Szynwałd (jetzt Bojków), als einen schlesischen inseldeutschen Dialekt (Wicherkiewicz 2013: 5).

$\mathrm{Zu}$ den jüngeren Erforschern des Wilmesaurischen zählen Rinaldo Neels aus Belgien, Alexander Andrason aus Island, Carlo Ritchie aus Australien sowie der jüngste Wilmesauer Muttersprachler und Verfechter der Revitalisierung: Tymoteusz Król. Neels schrieb in seiner Dissertation von 2012 u. a. über die Abstammung des Wilmesaurischen. Er vergleicht die phonologischen Systeme der westgermanischen Sprachen und kommt 
wie sein Vorgänger Kleczkowski zum Schluss, dass das Wilmesaurische typisch schlesische Merkmale mit diversen Eigentümlichkeiten aufweise (Ritchie 2012: 68, nach Wicherkiewicz 2013: 7-8). Über die Herkunft und die soziolinguistische Bedeutung des Wilmesauer Dialekts schrieb Wicherkiewicz mehrmals selbst.

\subsection{Das Wilmesaurische im 21. Jahrhundert}

Das 21. Jahrhundert brachte neue Entwicklungen für Wilmesau. Leider kann von einer erfreulichen Zukunft der winzig kleinen Sprachinsel kaum die Rede sein. Als 2007 der Studentische Germanistenzirkel am Institut für Germanische Philologie der Jagiellonen-Universität Krakau eine Reise ins ca. 70 km entfernte Wilmesau organisierte, lebten noch ca. hundert Sprecher des Wilmesaurischen. Allerdings waren die meisten in einem fortgeschrittenen Alter. Nach elf Jahren sank die Zahl der aktiven Sprecher des Wilmesaurischen auf ca. zwanzig Personen. Das ist das Ende für eine Sprache. Glücklicherweise fanden sich in Wilmesau einige junge Leute, die über die bilinguale Kompetenz des Wilmesaurischen verfügten, wie etwa der wichtigste Pfleger der lokalen Kultur, Sprache und Folklore Tymoteusz Król. Der Heimatverein Wilamowianie [Die Wilmesauer] pflegt Sprache und Volkstum. Als Ziel gibt der Verein an: „,...] die außergewöhnliche Kultur von Wilmesau zu pflegen, das im 13. Jahrhundert von den aus Flandern stammenden Siedlern gegründet wurde“ ${ }^{\text {"20 }}$. Die Kritik der Germanistik an der Flandern-Hypothese zeitigte aber auch gewisse Ergebnisse. Während des am 23. und 24. 2. 2018 in Wilmesau stattfindenden Muttersprachtages (Müterśspöhtag) wurde über den Mythos der flämischen Herkunft gesprochen, nicht mehr über eine Tatsache. Zum wichtigsten Ziel wurde jetzt die behördliche Anerkennung des Wilmesaurischen als Regionalsprache im Sinne der Europäischen Charta der Regional- oder Minderheitensprachen vom 5.11.1992. Bis dato ist dies nicht gelungen.

Vor dem wissenschaftlichen Hintergrund kann im Fall des Wilmesaurischen von einer niederländischen (,flämischen“), englischen oder gar schottischen Herkunft der Siedler, die man durch sprachliche Merkmale beweisen könne, keine Rede sein: Das Wilmesaurische ist ostmitteldeutsch. Dass manche Merkmale des Ostmitteldeutschen mit dem Niederdeutschen, Niederländischen und Englischen übereinstimmen, nicht aber mit der deutschen Standardsprache, ist kein Beweis für eine solche Herkunft. Dem [b]-Laut im Inlaut entspricht im Schlesischen [v], darum zyjwa (sieben), śtarwa (sterben), zytwer (Silber), tawa (leben). Der Wilmesauer Dialekt ist darüber hinaus konservativ und weist manche Merkmale des mittelalterlichen Deutsch auf, die einem Laien englisch oder niederländisch vorkommen mögen. Zum Beispiel ist die Wilmesauer Form kyng nicht vom englischen king entlehnt, sondern entspricht dem mittelhochdeutschen Wort künc. Die Form komp entspricht der mittelhochdeutschen Form kamp mit der schlesischen Verschiebung $a$ zu $o$, nicht unbedingt dem englischen comb. Die Wilmesauer Mundart wurde zu einer separaten westgermanischen Sprache erklärt. Das ist der Volkswille und daran ist nichts Verwerfliches, schließlich ist das Lëtzebuergesch auch durch Verschriftlichung eines mitteldeutschen Dialekts entstanden.

20 https://www.facebook.com/pg/StowarzyszenieWilamowianie/about/?ref=page_internal (03.12.2018). 
Attraktive Mythen sind aber nicht auszurotten und alles deutet darauf hin, dass die Fama die Sprache überleben wird. Eigentlich Schade - schließlich ist wohl eine echte schlesische Abstammung nicht schlechter als eine falsche flämische.

\section{ZUM AKTUELLEN GEBRAUCH DES WILMESAURISCHEN}

Obwohl die Sprechergemeinschaft des Wilmesaurischen im Aussterben begriffen ist, wird die Sprache von den jüngsten Sprechern gepflegt. Auch die offiziellen Stellen, wie das Stadtamt, verwenden sie, wenn auch nicht mehr als echtes Kommunikationsmittel, sondern als Lokalkolorit.

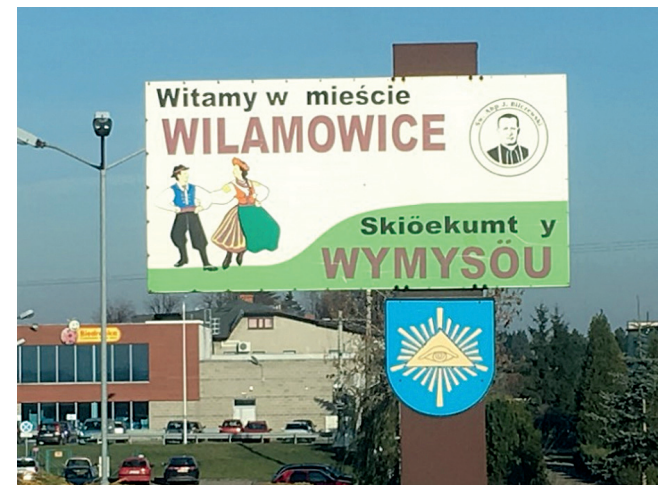

Abb. 2. Die Willkommenstafel beim Ortseingang mit zweisprachiger, polnisch-wilmesaurischen Inschrift.

Wilmesaurische Texte findet man auf Informationstafeln (Abb. 3) an Baudenkmälern, am neuen Denkmal des Stadtpatrons hl. Józef Bilczewski (Abb. 4) und an manchen Werbetafeln.

\begin{tabular}{|c|}
\hline 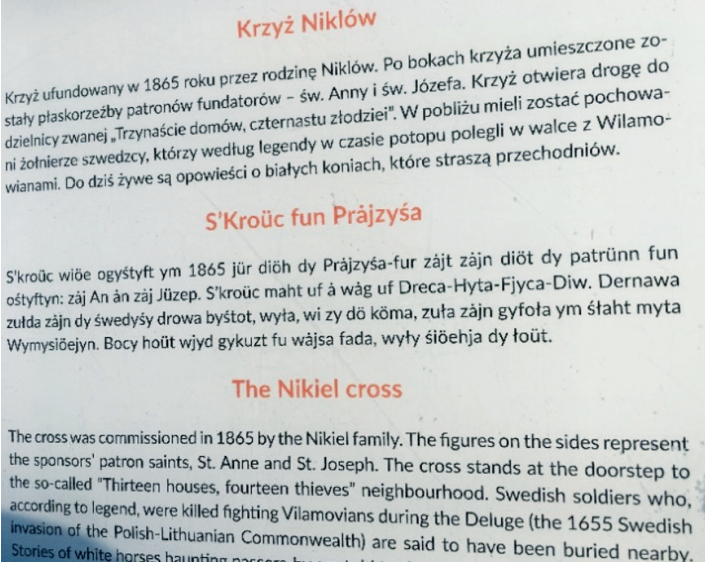 \\
\hline
\end{tabular}

Abb. 3: Die Sehenswürdigkeiten sind mit dreisprachigen, polnisch-wilmesaurisch-englischen Denkmaltafeln versehen. 


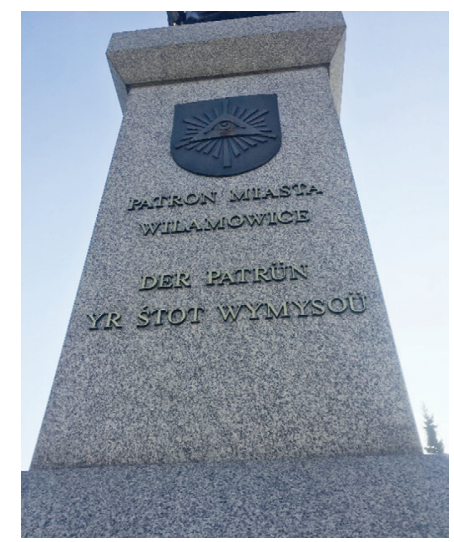

Abb. 4. Die Wilmesaurische Inschrift am Sockel des Denkmals des Stadtpatrons Józef Bilczewski.

Die jüngsten, bilingualen Sprecher des Wilmesaurischen benutzen es auch auf Facebook. ${ }^{21}$ Der bereits erwähnte Tymoteusz Król schreibt gerade seine Dissertation über die Revitalisierung einer Sprache am Beispiel des Wilmesaurischen. Die Revitalisierung ist ein interessantes Vorhaben, dessen Darstellung aber nicht in den Rahmen dieses Beitrags passt. Während früher eher von „flämisch“ die Rede war, scheint jetzt das Problem der Eigenständigkeit des Wilmesaurischen innerhalb der westgermanischen Sprachgruppe in den Vordergrund gerückt sein.

\section{Primärliteratur}

Metrika Baptisatorum ab ao (1641). Pfarrarchiv Wilamowice/Wilmesau.

Vragenlijsten met register (1931-1958). Bijdragen en mededelingen der DialectenCommissie van der Koninklijke Nederlandse Akademie van Wetenschapen te Amsterdam (1960). Bd. XXII, Amsterdam: N. V. Noord- Hollandsche Uitgevers Maatschappij.

\section{Sekundärliteratur}

BOCK, Friedrich (1916) Der Liega-Jirg und die Bielitzer Mundart. Teschen: Friedrich Bock.

BUKOWSKI, Jacob (1860) Gedichte in der Mundart der deutschen schlesisch-galizischen Gränzbewohner, resp. von Bielitz-Biala. Bielitz: Ludwig Zamarski.

CHROMIK, Grzegorz (2010) Schreibung und Politik. Untersuchungen zur Graphematik der frühneuhochdeutschen Kanzleisprache des Herzogtums Teschen. Kraków: Wydawnictwo Uniwersytetu Jagiellońskiego.

21 https://www.facebook.com/pg/StowarzyszenieWilamowianie/about/?ref=page_internal (03.12.2018). 
CHROMIK, Grzegorz (2016) „Sprachliche Analyse der Briefe kleinpolnischer Städte im Archiv von Bardejov/Bartfeld.“ In: R. Hünecke/S. Aehnelt (Hrsg.), Kanzlei und Sprachkultur. Beiträge der 8. Tagung des Arbeitskreises Historische Kanzleisprachenforschung, Dresden 3. bis 5. September 2015. Wien: Praesens Verlag, 107-118.

CHROMIK, Grzegorz (2017) „Zur Entwicklung der frühneuhochdeutschen und neuhochdeutschen Schriftsprache in der deutschen Sprachinsel Bielitz (Bielsko).“ In: S. Nefedov/L. Grigorieva/B. Bock (Hrsg.), Deutsch als Bindeglied zwischen Inlands- und Auslandsgermanistik. Beiträge zu den 23. GeSuS-Linguistik-Tagen in Sankt Petersburg, 22.-24. Juni 2015. Hamburg: Verlag Dr. Kovač, 173-182.

HUPPERT, Hugo (1976) Die angelehnte Tür. Bericht von einer Jugend. Halle/Saale: Mitteldeutscher Verlag.

KACPERCZYK, Ewa/Krystyna KORCZAK-ŻYDACZEWSKA (Hrsg.) (2013) Powierzchnia $i$ ludność $w$ przekroju terytorialnym w 2013 r. Warszawa: Główny Urząd Statystyczny. http://web.archive.org/web/20131220071949/http://www.stat. gov.pl:80/gus/5840_908_PLK_HTML.htm (15.09.2018).

KLECZKOWSKI, Adam (1921) Dialekt Wilamowic w zachodniej Galicji. Sktadnia (szyk wyrazów). Poznań: Gebethner i Wolff.

KUHN, Walter (1981) Geschichte der deutschen Sprachinsel Bielitz (Schlesien). Würzburg: Holzner Verlag.

LATOSIŃSKI, Józef (1909) Monografia Miasteczka Wilamowic. Kraków: Drukarnia Literacka.

MENZEL, Johann Joachim (1991) „Ius Teutonicum.“ In: Lexikon des Mittelalters (LexMA). 5. München/Zürich: Artemis-Verlag, 818.

MŁYNEK, Ludwik (1907) Narzecze wilamowickie (Wilhalmsauer Dialekt. Dy wymmysuaschy Gmoansproch). Tarnów: J. Pisz.

MOJMIR, Hermann (1930-1936) Wörterbuch der deutschen Mundart von Wilamowice. Kraków: Polska Akademia Umiejętności.

Morciniec, Norbert (1984) „Die flämische Ostkolonisation und der Dialekt von Wilamowice in Südpolen.“ Slavica Gandensia 11, 7-18.

REICHMANN, Oskar/Klaus-Peter WEGERA (1993) Frühneuhochdeutsche Grammatik. Tübingen: Niemeyer Verlag.

RÜCKERT, Heinrich (1878) Entwurf einer systematischen Darstellung der schlesischen Mundart im Mittelalter. Paderborn: Schöningh.

RYCKEBOER, Hugo (1984) „Die „Flamen“ von Wilamowice. Versuch zur Deutung einer bäuerlichen Überlieferung." Slavica Gandensia 11, 19-34.

STEDJE, Astrid (1989) Deutsche Sprache gestern und heute. München: Wilhelm Fink Verlag.

TANCER, Jozef/Anna GRUSKOVÁ (2018) „Sprechen Sie Karpatendeutsch.“ Dokumentarfilm über die karpatendeutsche Minderheit in der Slowakei. Regie: Jozef Tancer und Anna Grusková.

\section{Internetquellen:}

Bielsko Biała. http://bielskobiala.wyborcza.pl/bielskobiala/7,88025,21496653,of-jerwelt-przedstawienie-po-wilamowsku-na-deskach-teatru.html (26.11.2018). 
Biesik, Florian. Of jer wett. http://www.inne-jezyki.amu.edu.pl/Frontend/Text/Details/1455 (02.12.2018).

Biesik, Florian: Wymysau an wymysojer (nach: Wicherkiewicz Tomasz, Pochodzenie języka wilamowskiego - poglądy i badania, 2013) http:/www.revitalization.al.uw. edu.pl/eng/Wilamowicean?view $=8$ (15.09.2020).

Dziedzictwo Kulturowe Rzeczypospolitej. http://www.inne-jezyki.amu.edu.pl/Frontend/Language/Details/10 (04.12.2018).

ISO 639 Code Tables. https://iso639-3.sil.org/code_tables/639/data/w?page=1 (15.09.2018).

Gemeinde Wilmesau. https:/gmina.wilamowice.pl/strona/o-gminie (26.11.2018).

GLIŃSKI Mikołaj (2018) „Central Europe’s Most Mysterious Language.“ https://culture.pl/en/article/central-europes-most-mysterious-language (26.11.2018).

Lexer Online. http://woerterbuchnetz.de/cgi-bin/WBNetz/wbgui_py?sigle= Lexer\&mo$\mathrm{de}=$ Vernetzung\&lemid=LK02768\#XLK02768 (26.11.2018).

LinguisticEnclave in CentralEurope. https://news.ycombinator.com/item?id=17277691 (26.11.2018).

MORCINIEC, Norbert Vom Sterben einer Mundart. http://www.morciniec.eu/79,vom sterben_einer_mundart (03.12.2018).

Stowarzyszenie Wilamowianie https://www.facebook.com/pg/Stowarzyszenie Wilamowianie/about/?ref=page_internal (03.12.2018).

WICHERKIEWICZ, Tomasz (2013) Pochodzenie języka wilamowskiego - poglady

$i$ badania. http://www.revitalization.al.uw.edu.pl/Content/Uploaded/Documents/ wilamowski\%20jezyk\%20-\%20TW-4d5c8ee3-77f0-4807-bde2-9dd0198acc34.pdf (15.09.2018).

Wilamowice. https://de.wikipedia.org/wiki/Wilamowice (15.09.2018).

Wilamowice. https://en.wikipedia.org/wiki/Wilamowice (26.11.2018).

Wilmesaurisch. https://de.wikipedia.org/wiki/Wilmesaurisch (08.02.2020).

\section{Zusammenfassung \\ WILMESAU/WILAMOWICE - DIE NOCH EXISTENTE SPRACHINSEL IM SCHLESISCH-KLEINPOLNISCHEN GRENZRAUM UND DIE LEGENDE ÜBER IHREN NIEDERLÄNDISCHEN URSPRUNG}

Im Süden Polens liegt die Kleinstadt Wilamowice, deutsch Wilmesau, deren Einwohner einen germanischen Dialekt sprechen. Es handelt sich um eine schlesische Mundart des Deutschen aus der Gruppe der ostmitteldeutschen Dialekte, aber die meisten Einwohner und fast alle Polen sind von der flämischen Herkunft ihrer mittelalterlichen Vorfahren überzeugt. Dieser Beitrag verfolgt das Ziel, zu prüfen, inwiefern die mythenumwobene flämische Herkunft der Wahrheit entspricht. Es reicht jedoch nicht, die Ähnlichkeit der dortigen Mundart mit der deutschen und niederländischen Standardsprache zu vergleichen, man muss tiefer in die Vergangenheit greifen und sie mit älteren Perioden des Deutschen, insbesondere des Deutschen in Schlesien vergleichen. 
Dieser Vergleich zeigt weitgehende Ähnlichkeiten, die natürlich gegenüber dem Niederländischen nicht vorhanden sind. Vor allem trennt die im Wilmesaurischen durchgeführte 2. Lautverschiebung diese Mundart vom Niederländischen, wenn auch der Durchführungsgrad insbesondere im Bereich der Verschiebung von $\mathrm{p}$ zu pf nicht so stark ist, wie in der deutschen Standardsprache (wilmesaurisch: töp 'Topf', troppa 'Tropfen', fund ,Pfund'). Der Mythos erweist sich als ein attraktiver, exotischer Mythos ohne jegliche wissenschaftliche Begründung. Mittlerweile ist man sogar bemüht, die Mundart als separate germanische Mikrosprache anerkennen zu lassen. Schade nur, dass dieser Dialekt vom Aussterben bedroht ist, da die Zahl der aktiven Sprecher zur Zeit circa zwanzig beträgt.

Schlüsselwörter: Sprachinsel, Schlesisch, Bielitz, Wilamowice/Wilmesau

\author{
Abstract \\ WILMESAU/WILAMOWICE - LANGUAGE ENCLAVE \\ IN THE BORDER REGION OF SLESIA-LESSER POLAND AND \\ THE LEGEND OF ITS DUTCH ORIGIN
}

In the south of Poland, there is a small town called Wilamowice (or Wilmesau in German) whose residents speak a dialect of German, specifically the Silesian dialect of German. Although it is a dialect of East Central German, it is often believed that its speakers have medieval ancestors with Flemish roots. The aim of the article is to verify how true this belief might be. It is, however, not enough to compare this dialect with standard German or standard Dutch. A deep dive into the past is needed so that the dialect in question can be examined against the characteristics of German (particularly Silesian German) in its various historical phases. Such a comparison reveals considerable similarities with Silesian German, and not Dutch. In particular, what makes the Wilamowice dialect different from Dutch dialects is the second Germanic consonant shift, although here it was not as strong as it was in Standard German, especially as far as the change from $p$ to $p f$ is concerned (Wilamowice dialect: töp, German Topf, Wilm. Troppa, Germ. Tropfen, Wilm. Fund, Germ. Pfund). The supposed Dutch connection is, therefore, an attractive, exotic myth with no scientific basis. Currently, attempts are made to classify the Wilamowice dialect as a separate German microlanguage. Unforunately, the dialect is in danger of extinction, as the number of its active users has fallen to about twenty.

Keywords: language island, Silesian German, Bielitz, Wilamowice/Wilmesau 


\section{Povzetek \\ WILAMOWICE (NEM. WILMESAU) - JEZIKOVNI OTOK V ŠLEZIJSKO- MALOPOLJSKEM MEJNEM PROSTORU IN LEGENDA O NJEGOVEM NIZOZEMSKEM IZVORU}

Na jugu Poljske leži manjše mesto Wilamowice (nem. Wilmesau), njegovi prebivalci pa govorijo germansko narečje. Gre za šlezijsko narečje iz skupine vzhodnosrednjenemških narečij, pri večini prebivalcev in skorajda vseh Poljakih pa prevladuje prepričanje, da so njihovi srednjeveški predniki flamskega izvora. Cilj prispevka je ugotoviti, v kolikšni meri je mit o flamskem izvoru utemeljen. Pri tem ni dovolj zgolj primerjati tamkajšnji narečni govor z nemškim ter nizozemskim knjižnim jezikom, temveč se je potrebno ozreti tudi globlje v preteklost in ga primerjati s starejšimi obdobji nemščine, še posebej s šlezijsko nemščino. Primerjava pokaže na precejšnje podobnosti z nemščino, ki jih v primerjavi z nizozemščino ni mogoče zaznati. Narečni govor Wilamowic ločuje od nizozemščine drugi premik glasov, čeprav stopnja premika, še posebej pri glasu $p$ v pf, ni tako visoka, kot jo je doživela nemška knjižna nemščina (wilm: töp, nem. Topf 'lonec', wilm. troppa, nem. Tropfen 'kaplja', wilm. fund, nem. Pfund, 'funt'). Pri flamskem mitu gre le za privlačen, eksotičen mit brez kakršnekoli znanstvene podlage. V sedanjem času obstajajo celo poskusi, da bi bil ta govor priznan kot poseben germanski mikrojezik, ob vsem tem pa je škoda, da to narečje izumira, saj ga trenutno aktivno uporablja le še približno dvajset govorcev.

Ključne besede: jezikovni otok, šlezijska nemščina, Bielitz/Belsko, Wilamowice/Wilmesau 